\title{
REATOR DE UV-OZÔNIO COM LÂMPADA A VAPOR DE MERCÚRIO A ALTA PRESSÃO MODIFICADA PARA TRATAMENTO SUPERFICIAL DE ÓXIDOS TRANSPARENTES CONDUTIVOS UTILIZADOS EM DISPOSITIVOS POLIMÉRICOS ELETROLUMINESCENTES
}

\author{
Emerson Roberto Santos*, Fábio Conte Correia e Shu Hui Wang \\ Departamento de Engenharia Metalúrgica e de Materiais, Escola Politécnica, Universidade de São Paulo, 05508-900 São Paulo - SP, Brasil \\ Pilar Hidalgo e Fernando Josepetti Fonseca \\ Departamento de Engenharia de Sistemas Eletrônicos, Escola Politécnica, Universidade de São Paulo, 05508-970 São Paulo - SP, Brasil \\ Elvo Calixto Burini Júnior e Adnei Melges de Andrade \\ Instituto de Eletrotécnica e Energia, Universidade de São Paulo, 05508-010 São Paulo - SP, Brasil
}

Recebido em 8/7/09; aceito em 29/3/10; publicado na web em 9/8/10

\begin{abstract}
UV-OZONE REACTOR WITH MODIFIED HIGH PRESSURE MERCURY VAPOR LAMP FOR SURFACE TREATMENT OF TRANSPARENT CONDUCTIVE OXIDES USED IN ELECTROLUMINESCENT POLYMERIC DEVICES. An UV-Ozone reactor was developed with an ignition tube extracted into HID mercury lamp used to irradiation on zinc oxide $(\mathrm{ZnO})$ and fluorinated tin oxide (FTO) films for PLEDs devices. Different exposures times were used. In contact angle measurements revealed better results for $\mathrm{ZnO}$ and FTO by 15 and 5 min, respectively. In Diffuse Reflectance Infra-red Fourier Transformed (DRIFT) spectroscopy allowed the observation of water, hydrocarbon and carbon dioxide adsorbed on the untreated TCO surfaces. After the UV-Ozone treatment the contaminants were significantly reduced or eliminated and the PLEDs devices decreased threshold voltages in comparison with respectively untreated TCOs.
\end{abstract}

Keywords: UV-Ozone; HID mercury vapor lamp; transparent conductive oxide.

\section{INTRODUÇÃO}

Óxidos transparentes condutores (TCOs) são filmes semicondutores utilizados como eletrodo anodo na construção de dispositivos poliméricos eletroluminescentes, conhecidos por P/OLEDs (diodos poliméricos/orgânicos emissores de luz). Estes filmes possuem características ópticas e elétricas favoráveis para um bom desempenho dos dispositivos, tais como: boa transparência na faixa de comprimento de onda da luz visível, banda proibida entre 4,4 4,8 eV, baixa resistividade elétrica, estabilidade química e fácil decapagem. Na montagem de P/OLEDs, a etapa inicial pode ocorrer com um tratamento superficial sobre o TCO, eliminando contaminantes indesejáveis das superfícies como, por exemplo, umidade, hidrocarbonetos e gás carbônico, que prejudicam o bom desempenho dos dispositivos. ${ }^{1}$ Neste caso, a técnica de tratamento de superfícies com UV-Ozônio pode ser utilizada em TCOs depositados sobre substratos de vidro e também plástico. ${ }^{2}$ Devido à necessidade de protocolos de preparação de dispositivos poliméricos eletroluminescentes mais eficientes, tem-se tornada necessária a utilização de métodos de limpeza superficial mais simples e reprodutivos, com procedimentos mais eficazes e com custo reduzido. A limpeza utilizando a radiação com UV-Ozônio tem sido empregada com grande sucesso na área da microeletrônica, desde a década de 70. ${ }^{3}$ Equipamentos de UV-Ozônio comerciais possuem uma lâmpada elétrica com gás de mercúrio, denominada lâmpada de catodo frio. ${ }^{4}$ Este tipo de lâmpada fornece radiação ultravioleta, gerando ozônio. Na literatura, tem sido reportado que para a produção do ozônio é necessária a radiação ultravioleta baixa (de 100 até $280 \mathrm{~nm}$ ) com um comprimento de onda $\lambda<243 \mathrm{~nm}$, que dissocia a molécula de oxigênio proveniente do ar atmosférico. Outro comprimento de onda entre 240 e $320 \mathrm{~nm}$ causa a dissociação da molécula de ozônio liberando radicais livres de oxigênio, que atuam efetivamente no processo de tratamento dos TCOs. Este método descrito pode ser explicado com quatro equações químicas (de 1 até 4 ), no ciclo de Sidney Chapman: ${ }^{5}$

\footnotetext{
*e-mail: emerson@lme.usp.br
}

Formação do ozônio

$\mathrm{O}_{2}($ ar atmosférico $)+\mathrm{h} v(\lambda<243 \mathrm{~nm}) \rightarrow 2 \mathrm{O} \cdot$ (radicais livres) $(1)$

$\mathrm{O} \cdot+\mathrm{O}_{2} \rightarrow \mathrm{O}_{3}$ (ozônio)

Dissociação do ozônio

$\mathrm{O}_{3}+\mathrm{hv}(240<\lambda<320 \mathrm{~nm}) \rightarrow \mathrm{O}_{2}+\mathrm{O}^{\bullet}$

$\mathrm{O} \cdot+\mathrm{O}_{3} \rightarrow 2 \mathrm{O}_{2}$

Na técnica de UV-Ozônio, devido ocorrer a geração da radiação ultravioleta e também produção de ozônio (que é um forte agente oxidante) ambos potencialmente prejudiciais à saúde humana, esses tipos de lâmpadas devem ser isoladas em ambiente fechado como, por exemplo, no interior de câmaras. ${ }^{6}$

A condutividade elétrica dos TCOs, por exemplo, filmes de ITO (óxido de índio e estanho), pode ser afetada pela deficiência de ligações de oxigênio sobre a superfície. ${ }^{7}$ Nesse caso, o tratamento com UV-Ozônio produzido por uma lâmpada a vapor de mercúrio a alta pressão modificada é capaz de gerar radicais livres de oxigênio para completar as ligações químicas superficiais, contribuindo também com melhor adesão de polímeros. Esse efeito é capaz de produzir diferenças significantes no desempenho final do dispositivo, como a redução da tensão de limiar e/ou aumento da luminância em P/ OLEDs, desempenho que tem sido reportado na literatura utilizando filmes de ITO$^{2}$.

Neste trabalho é estudado o efeito do tratamento de UV-Ozônio utilizando outros tipos de TCOs, como fonte alternativa em substituição aos filmes de ITO, que possuem custo comercial elevado. Filmes de FTO (óxido de estanho dopado com flúor) e ZnO (óxido de zinco) depositados sobre substratos de vidro foram testados em dispositivos PLEDs, com o intuito de avaliar os seus respectivos comportamentos no desempenho final desses tipos de dispositivos.

Os filmes de $\mathrm{ZnO}$ foram fabricados pela técnica de sputtering, apresentaram resistência de folha de 90-120 $\Omega$ / e foram produzidos 
no laboratório do Centro de Investigações de Materiais (CENIMAT), Faculdade de Ciências e Tecnologia, Universidade Nova de Lisboa, Portugal. ${ }^{8}$ Os filmes de FTO foram produzidos pela técnica de spray pyrolysis, apresentaram resistência de folha de 6-8 $\Omega / \square$ e foram fabricados pela empresa brasileira Flexitec Eletrônica Orgânica Ltda. ${ }^{9}$

\section{LÂMPADA A VAPOR DE MERCÚRIO A ALTA PRESSÃo}

Para a produção da radiação ultravioleta e ozônio, foi utilizada uma lâmpada a vapor de mercúrio a alta pressão com longo tempo de vida, acima de 29.000 h; é de fácil manuseio, preço reduzido e apresenta um tubo de ignição interno contendo vapor de mercúrio. ${ }^{10}$ A parede interna do bulbo externo desse tipo de lâmpada é coberta com uma camada de fósforo, para bloquear a emissão de raios ultravioleta. No interior dessa lâmpada existe outro bulbo, chamado de tubo de ignição, que é preenchido com argônio. Dependendo da lâmpada, pode ter um ou dois eletrodos para partidas e repartidas rápidas. ${ }^{11}$ Este tipo de lâmpada ainda é muito utilizada em postes de iluminação pública (apresentando emissão de cor branco-azulada).

\section{PARTE EXPERIMENTAL}

Nos tratamentos, foi utilizada uma lâmpada a vapor de mercúrio a alta pressão de $400 \mathrm{~W}$ com rosca tipo Edison (modelo E 40). O tubo de ignição foi rosqueado em um porta-lâmpada no interior de um refletor de alumínio com volume aproximado de $13 \mathrm{~L}$. Dois pequenos ventiladores foram adaptados próximos à fonte de radiação UV. Durante os experimentos, o refletor (reator) foi colocado de cabeça para baixo sobre uma base de madeira coberta com folha de papel alumínio. Para melhorar a vedação do reator sobre a base, em toda a borda do refletor foi encaixado um anel de borracha.

Foram monitoradas a concentração de ozônio e a temperatura no interior do reator ao longo da operação. Para o monitoramento da concentração de ozônio, foi utilizada um tubo de teflon, com diâmetro de $2 \mathrm{~mm}$. A extremidade da sonda foi posicionada a uma distância de $2 \mathrm{~mm}$ do tubo de ignição e conectada diretamente ao monitor de ozônio (In DevR 2B Technologies Dual Beam, modelo 205). Para registrar a temperatura, foi utilizado um termômetro (Minipa, modelo ET-2882) conectado a um termopar tipo K (Cromel/Alumel, que cobre uma faixa entre $\approx-200 \mathrm{e} \approx 1.300^{\circ} \mathrm{C}$ ), também com distância de $2 \mathrm{~mm}$ da fonte de UV.

Foram preparadas amostras dos substratos com filmes de FTO e $\mathrm{ZnO}$ depositados sobre substratos de vidro com dimensão de 1 x $1 \mathrm{~cm}$ para caracterização e de 2,5 x $2,5 \mathrm{~cm}$ para a preparação de dispositivos. As amostras foram previamente limpas por imersão, primeiro em acetona durante $30 \mathrm{~min}$ e depois em álcool isopropílico também durante $30 \mathrm{~min}$, ambos os procedimentos em ultrassom e, finalmente, as amostras foram secas em jato de nitrogênio.

Durante os tratamentos de UV-Ozônio, os TCOs foram posicionados no centro do reator e irradiados em tempos distintos. Para comparação dos resultados, amostras sem tratamentos foram utilizadas como referência.

Medições de ângulo de contato foram realizadas utilizando-se equipamento da marca Tantec, modelo Cam-Micro. Nessa técnica, com uma microsseringa colocou-se uma gota de poli(3,4-etilenodioxitiofeno) com poli(estirenossulfonato) ou PEDOT:PSS. A sombra da gota, obtida com um sistema de iluminação, foi projetada sobre um anteparo contendo um transferidor. $\mathrm{O}$ ângulo interno da gota foi medido diretamente em graus, tendo como referência o plano horizontal. Para analisar compostos ou elementos químicos adsorvidos sobre as superfícies dos TCOs foi utilizada a técnica de infravermelho por transformada de Fourier com refletância difusa (DRIFT). Essas medições foram realizadas no equipamento da marca Thermo
Fisher Scientific Nicolet, modelo Magna-IR 560 e foram detectadas espécies ligadas química ou fisicamente à superfície, identificadas pelas respectivas bandas de absorção. ${ }^{12}$ Nessa técnica de DRIFT, um feixe de infravermelho incide diretamente sobre a superfície do TCO interagindo com os elementos químicos ou compostos, ocorrendo o espalhamento da radiação infravermelha. Uma parte desta radiação é refletida da amostra (e que pode ocorrer em qualquer ângulo) e capturada por um espelho côncavo, sendo retransmitida diretamente ao sensor detector. Nos experimentos foram obtidas 128 varreduras (valor máximo fornecido pelo software do equipamento) durante alguns segundos para cada medição, sendo obtida a média aritmética para cada ponto registrado no gráfico de absorção em função do número de onda. Espectros de transmitância das amostras foram obtidos na região UV-VIS, utilizando o equipamento Shimadzu modelo PC-1650. Nesta técnica utilizou-se o ar ambiente como referência durante as medições.

Após as irradiações, dado que as superfícies dos filmes se tornam reativas adsorvendo elementos químicos e compostos do próprio ar atmosférico ambiente, foram depositados imediatamente, pela técnica de spin-coating, os filmes poliméricos com PEDOT:PSS, utilizados nos dispositivos como camada transportadora de lacunas (HTL). As espessuras formadas nesses filmes foram de $\approx 56 \mathrm{~nm}$ (com os parâmetros de processos em $3.000 \mathrm{rpm}$ por $30 \mathrm{~s}$ ). Após esse procedimento, as amostras foram levadas até uma estufa a vácuo, para a remoção do solvente, permanecendo em $100{ }^{\circ} \mathrm{C}$ durante $10 \mathrm{~min}$. Em seguida, sobre o filme de PEDOT:PSS, foi depositado o polímero emissor com $\mathrm{OC}_{1} \mathrm{C}_{10}$-PPV (poli (2-metoxi-5-(3-,7-dimetil-octiloxi)-1,4-fenileno vinileno) dissolvido em clorofórmio, com concentração de $7 \mathrm{mg} / \mathrm{mL}$. A espessura do filme, resultante de deposição a $3.000 \mathrm{rpm}$, por 60 $\mathrm{s}$, foi de $\approx 190 \mathrm{~nm}$. Após essa etapa de deposição as amostras foram levadas novamente para a estufa em vácuo, com temperatura de 55 ${ }^{\circ} \mathrm{C}$ durante $120 \mathrm{~min}$, para remoção do solvente. Terminada esta etapa, as amostras foram carregadas no interior de uma metalizadora para a deposição do eletrodo catodo metálico, utilizando alumínio, pela técnica de evaporação térmica em vácuo.

\section{RESULTADOS E DISCUSSÃO}

As medições de concentração de ozônio e temperatura foram registradas simultaneamente, a cada $2 \mathrm{~min}$. Foram realizados dois experimentos distintos, com e sem ventilação no interior do reator. Com ventilação, foi observado um aumento rápido da concentração de ozônio durante os primeiros $10 \mathrm{~min}$, apresentando $\approx 60$ ppm e, após esse período, foi observada uma diminuição menos acentuada até o tempo final de $60 \mathrm{~min}, \mathrm{com} \approx 30 \mathrm{ppm}$. Sem ventilação, verificou-se um pico máximo da concentração de ozônio até os primeiros 2 min, atingindo $\approx 30 \mathrm{ppm}$. Após esse período, verificou-se uma diminuição considerável da produção de ozônio até 10 min de funcionamento do reator. A possível explicação para as elevações encontradas nas concentrações de ozônio pode estar relacionada à disponibilidade de oxigênio no interior do reator, que é consumido durante os sucessivos ciclos da dissociação do ozônio (formação do ozônio pelo ciclo de Sidney Chapman), enquanto a lâmpada ainda está sendo aquecida.

A Figura 1 apresenta os resultados das medições de concentração de ozônio em função do tempo, utilizando-se o reator de UV-Ozônio, com e sem ventilação.

Nos experimentos realizados, verificou-se a influência no resfriamento da lâmpada devido à ação dos ventiladores, que tende a homogeneizar a temperatura no interior da câmara. A Figura 2 apresenta a evolução da temperatura em função do tempo, utilizando-se o reator de UV-Ozônio, com e sem ventilação.

Em ambos experimentos, se compararmos os dois picos da concentração de ozônio relacionados com os dois comportamentos das 


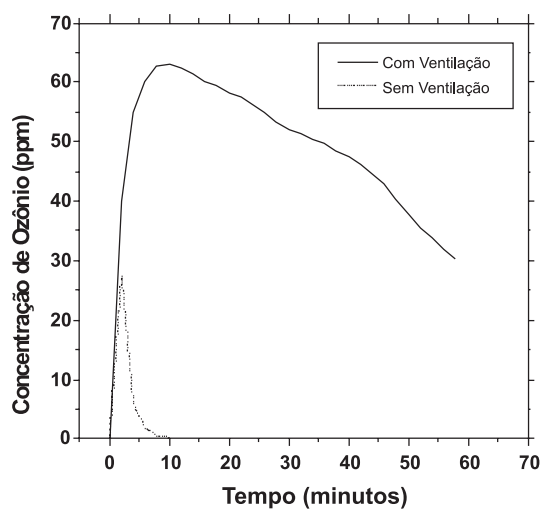

Figura 1. Concentração de ozônio em função do tempo no interior do reator de UV-Ozônio, com e sem ventilação

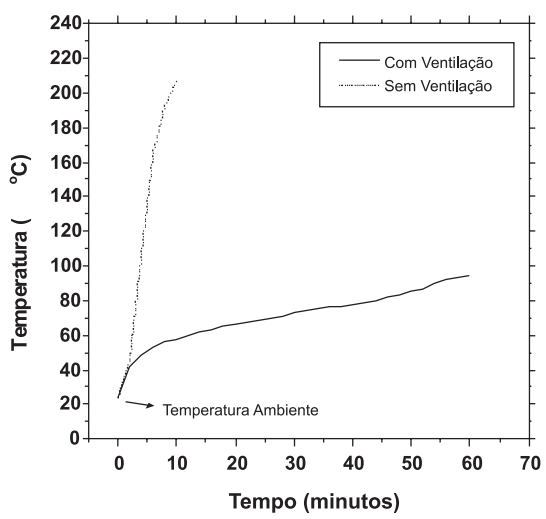

Figura 2. Temperatura em função do tempo no interior do reator de UVOzônio, com e sem ventilação

temperaturas, verifica-se que o maior pico da concentração de ozônio com ventilação ocorreu $\mathrm{em} \approx 60{ }^{\circ} \mathrm{C}$ durante os primeiros $10 \mathrm{~min} \mathrm{e}$ sem ventilação ocorreu em $\approx 50^{\circ} \mathrm{C}$ durante os primeiros $2 \mathrm{~min}$, sendo que o pico da concentração máxima de ozônio produzido com ventiladores corresponde praticamente ao dobro daquela observada sem ventiladores. Se compararmos a lâmpada a vapor de mercúrio a alta pressão modificada, utilizada nesse experimento, com dados reportados na literatura sobre lâmpadas de vapor de mercúrio a baixa pressão (germicidas), verifica-se que nestas a eficiência de $100 \%$ dos raios ultravioleta está associada à temperatura no bulbo, sendo encontrada a temperatura de $\approx 40^{\circ} \mathrm{C}$; porém, em ambos os casos, possivelmente o vidro utilizado ainda pode atenuar a produção dos raios ultravioleta. ${ }^{13}$

Antes dos tratamentos foram realizadas medições das transmitâncias dos filmes de ZnO e FTO por espectroscopia UV-VIS. Sabendose previamente que o maior comprimento de onda de intensidade na emissão apresentado pelo polímero emissor com $\mathrm{OC}_{1} \mathrm{C}_{10}$-PPV é de $\approx 590 \mathrm{~nm}$, nesse mesmo comprimento de onda foi encontrada a transmitância para o filme de FTO com $\approx 82 \%$ e para os filmes de $\mathrm{ZnO}$ com $\approx 93 \%$, ou seja, foi observada uma diferença de $\approx 11 \%$ entre ambos os filmes. Verifica-se que o valor de transmitância apresentado pelo filme do $\mathrm{ZnO}$ está mais próximo do valor apresentado também para o filme de ITO com $\approx 93 \%$. $^{14}$

Nas medições de ângulo de contato foram utilizadas 3 amostras para cada tempo de tratamento com 5,10 e 15 min e, também, amostras sem tratamento utilizando os dois tipos de TCOs. A média aritmética referente a cada 3 amostras (para cada tempo referido) juntamente com o desvio padrão foram obtidos. As amostras tratadas revelaram mudanças significativas em comparação às amostras sem tratamento. Foi possível observar em todas as amostras que a gota de solução aquosa com o polímero PEDOT:PSS se espalhou melhor, diminuindo o ângulo de contato sobre os filmes de FTO tratados com 5 min e $\mathrm{ZnO}$ com $15 \mathrm{~min}$. A Tabela 1 apresenta os resultados de ângulo de contato para ambos TCOs tratados em diferentes tempos, incluindo a amostra sem tratamento.

Tabela 1. Ângulo de contato para os filmes de FTO e $\mathrm{ZnO}$ tratados e sem tratamento

\begin{tabular}{ccc}
\hline Tempo de tratamento $(\mathrm{min})$ & FTO grau $\left({ }^{\circ}\right)$ & $\mathrm{ZnO}$ grau $\left({ }^{\circ}\right)$ \\
\hline 5 & $\mathbf{2 0} \pm \mathbf{1}$ & $40 \pm 3$ \\
10 & $37 \pm 1$ & $31 \pm 1$ \\
15 & $43 \pm 1$ & $\mathbf{2 0} \pm \mathbf{1}$ \\
Sem tratamento & $32 \pm 3$ & $43 \pm 1$ \\
\hline
\end{tabular}

Devido aos filmes tratados apresentarem melhores resultados de molhabilidade por ângulo de contato com FTO/5 min e $\mathrm{ZnO} / 15$ min, estes mesmos TCOs com os referidos tempos foram utilizados nas caracterizações com espectroscopia de DRIFT. Antes da aquisição dos espectros de DRIFT, foi realizada a aquisição de um background (correção de linha de fundo), para eliminar o efeito da umidade e $\mathrm{CO}_{2}$ do ambiente. Nessa técnica, uma amostra de cada TCO foi medida primeiramente sem tratamento e, logo em seguida, tratada e novamente medida para manter o efeito do tratamento, uma vez que sobre as superfícies dos TCOs pode haver perda de oxigênio ao longo do tempo. ${ }^{15}$ Os resultados de DRIFT apresentados com espectros de absorbância em função do número de onda, são mostrados na Figura 3 para os filmes de FTO e na Figura 4 para os filmes de $\mathrm{ZnO}$.

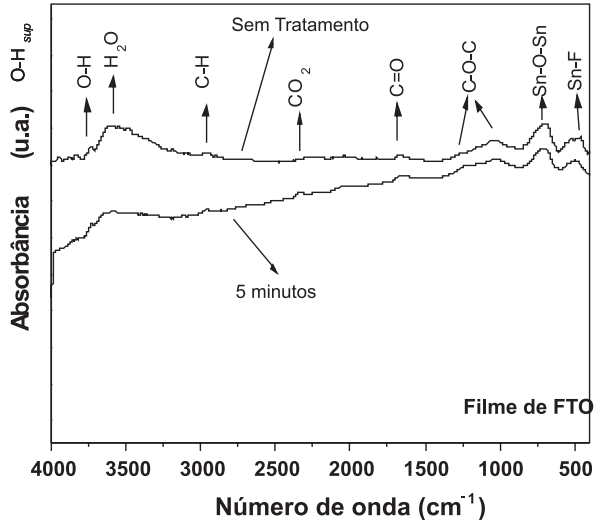

Figura 3. Espectros de absorbância de DRIFT dos filmes de FTO sem tratamento e com 5 min de tratamento

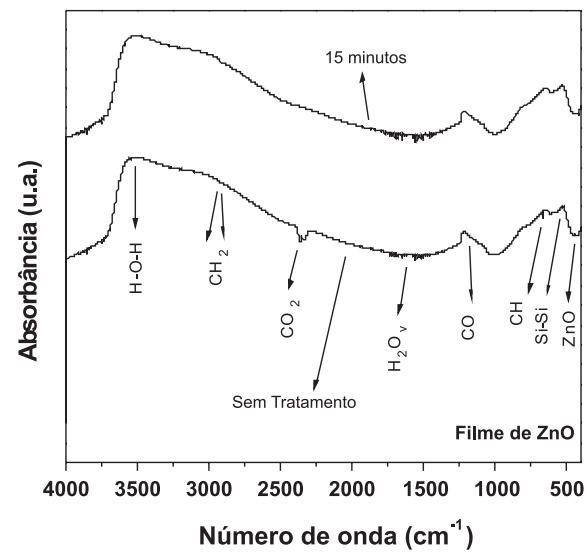

Figura 4. Espectros de absorbância de DRIFT dos filmes de ZnO sem tratamento e com 15 min de tratamento 
As curvas médias contidas nas Figuras 3 e 4 encontram-se deslocadas, intencionalmente, em relação ao eixo das ordenadas para facilitar a visualização. Portanto, não permitem comparação além de variação relativa da absorbância para mesmo valor de número de onda.

Em termos gerais, os resultados das medições por espectroscopia de DRIFT para as amostras de FTO revelaram uma diminuição considerável dos grupos $\mathrm{O}-\mathrm{H}, \mathrm{H}_{2} \mathrm{O}$ e $\mathrm{C}-\mathrm{H}$ sobre a superfície, após o tratamento de $5 \mathrm{~min}$. Possivelmente a diminuição do grupo O-H deva ser atribuída ao próprio ambiente, uma vez que também foi encontrada na mesma amostra ainda sem tratamento. O que se verificou foi que esse filme revelou ser susceptível ao tratamento oxidativo de UV-Ozônio, causando modificações consideráveis sobre a sua superfície. Comparando com a película de $\mathrm{ZnO}$ tratada com $15 \mathrm{~min}$ foram verificadas, principalmente, as diminuições de $\mathrm{CO}_{2}$ e grupos $\mathrm{C}-\mathrm{H}$, sendo que esse decréscimo do grupo $\mathrm{C}-\mathrm{H}$ deve ser atribuído a alguma substância gordurosa.

As bandas de absorção desses grupos apresentaram-se com intensidades mais reduzidas no espectro do filme de $\mathrm{ZnO}$ quando comparadas com o FTO. Essa diminuição parcial do $\mathrm{CO}_{2}$ adsorvido também foi verificada anteriormente em filmes de ITO tratados pelo mesmo método. ${ }^{2}$ Portanto, o que ficou esclarecido é que o tratamento nas superfícies dos substratos é eficiente em função do tempo e mostrou uma superfície mais inerte ou menos contaminada utilizando ambos os TCOs.

Em relação aos dispositivos, todos foram montados no mesmo instante e nas mesmas condições de pressão e temperatura ambiente. Utilizando os dois tipos de TCOs foram construídos e polarizados 6 PLEDs (sem encapsulamento) sobre um mesmo substrato e selecionado apenas o dispositivo com TCO tratado e não tratado que apresentou melhor desempenho, ou seja, a menor tensão de limiar. Nos gráficos de $\mathrm{I}-\mathrm{V}$, as tensões de limiar dos dispositivos foram determinadas traçando retas tangentes a partir dos valores máximos das curvas, até atingir o eixo da tensão, como proposto por Lee et al.. ${ }^{16}$ Uma pequena diferença no valor da tensão de limiar foi encontrada para os dispositivos com filmes de $\mathrm{ZnO}$ tratados com 15 min em comparação com a amostra sem tratamento, na qual se encontraram os valores de $\approx 9 \mathrm{e} \approx 10,5 \mathrm{~V}$, respectivamente, como mostrado na Figura 5.

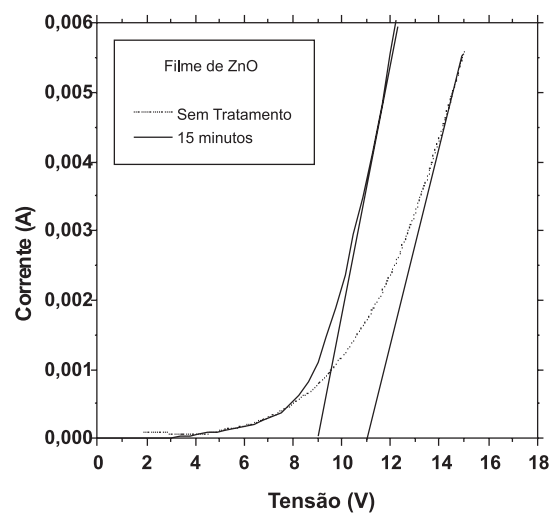

Figura 5. Curvas de I-V dos dispositivos PLEDs construídos com os filmes de $\mathrm{ZnO}$ sem tratamento e com 15 min de tratamento

Nos PLEDs montados com filmes de FTO, a comparação utilizando o mesmo método para se obter a tensão de limiar nas curvas I-V foi mais pronunciada para a amostra tratada com 5 min apresentando uma tensão de limiar de $\approx 7 \mathrm{~V}$, como mostrado na Figura 6 .

As curvas de I-V dos dispositivos permitem observar que filmes de FTO sem tratamento resultaram em uma curva instável, dificultando a obtenção mais aproximada do valor da tensão de limiar. Observa-se que o PLED montado com FTO tratado com UV-Ozônio apresenta

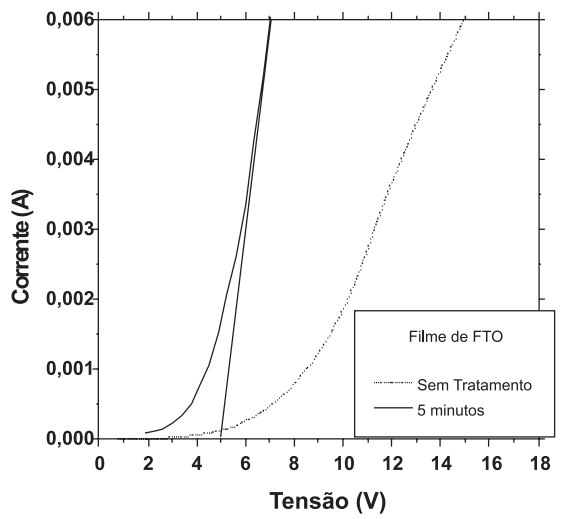

Figura 6. Curvas de I-V dos dispositivos PLEDs construídos com os filmes de FTO sem tratamento e com 5 min de tratamento

um desempenho bastante satisfatório, reforçando a relevância do tratamento no desempenho dos dispositivos eletroluminescentes construídos com este tipo de TCO. Comparando os desempenhos apresentados pelos filmes de FTO e ZnO nos dispositivos, ambos tratados na melhor condição, pode-se observar que a tensão de limiar do dispositivo montado com FTO foi menor, embora tenha sido verificado que o filme de FTO apresentou transmitância menor que o filme de $\mathrm{ZnO}$.

\section{CONCLUSÃO}

O procedimento de tratamento de TCOs utilizando o reator de UV-Ozônio com uma lâmpada a vapor de mercúrio a alta pressão modificada mostrou-se bastante eficiente com a adição de dois ventiladores, sendo adequado para a preparação de PLEDs, reduzindo contaminantes sobre as superfícies. Comparando os espectros de DRIFT das amostras tratadas e sem tratamento, foi possível verificar que o tratamento propiciou a diminuição dos grupos O-H e C-H da superfície dos filmes de FTO e de $\mathrm{CO}_{2}$ e grupos C-H da superfície do filme de $\mathrm{ZnO}$. Os resultados das medições de ângulo de contato mostraram maior molhabilidade da solução do polímero semicondutor PEDOT:PSS sobre os filmes tratados de $\mathrm{ZnO}$ por 15 min e FTO por $5 \mathrm{~min}$. As curvas de I-V dos dispositivos PLEDs, produzidos com TCOs tratados, apresentaram curvas típicas de diodo emissor de luz. Dispositivos construídos com FTO tratado apresentaram melhor desempenho, com menor tensão de limiar, comparativamente aos produzidos com filmes de $\mathrm{ZnO}$ e, na melhor condição de tempo de tratamento utilizado em cada TCO, observou-se uma diferença de $\approx 4 \mathrm{~V}$ na tensão de limiar, revelando uma significativa melhora no desempenho de dispositivos construídos com FTO.

\section{REFERÊNCIAS}

1. Li, C. N.; Kwong, C. Y.; Djurisic, A. B.; Lai, P. T.; Chui, P. C.; Chan, W. K.; Liu, S. Y.; Thin Solid Films 2005, 2, 57.

2. Santos, E. R.; Correia, F. C.; Junior, E. C. B.; Wang, S. H.; Yamasoe, M. A.; Hidalgo P.; Fonseca, F. J.; Andrade, A. M.; Sens. \& Trans. 2009, $101,12$.

3. Vig, J. R.; Le Bus, J. W.; IEEE Trans. PHP 1976, 12, 365.

4. UVP Inc., Operating instruction manual of PR-100 model, 2002.

5. Chapman, S.; Mem. Roy. Met. Soc. 1930, 3, 103.

6. Diffey, B. L.; Methods 2002, $28,4$.

7. Lin, H.-N.; Chen, S.-H; Perng, G.-Y.; Chen, S.-A.; Appl. Phys. 2001, 89, 3976.

8. Nunes, P.; Fernandes, B.; Fortunato, E.; Vilarinho, P.; Martins, R.; Thin Solid Films 1999, 337, 176. 
9. Canestraro, C. D.; Oliveira, M. M.; Valaski, R.; Persson, C.; Silva, M. V. S.; David, D.; Pepe, I.; Silva, A. F.; Roman, L. S.; Persson, C.; Appl. Surf. Sci. 2008, 255, 1874.

10. Sylvania Light. Int.; Catalogue of HSL-BW 400W, E40 model 2005.

11. DRAFT TSD; HID Lamps Analysis of Potential Energy Savings, 2004.

12. Falla, M. D. P. H.; Tese de Doutorado, Universidade de São Paulo, Brasil, 2005.

13. Philips UV Disinfection - Application Information, 2004.
14. Santos, E. R.; Tese de Doutorado, Universidade de São Paulo, Brasil, 2009.

15. Berntsen, A.; Croonen, Y.; Liedenbaum, C.; Schoo, H.; Visser, R.-J.; Vleggaar, J.; Weijer, P. V.; Opt. Mater. 1998, 9, 125.

16. Lee, W. J.; Fang, Y.-K.; Ho, J.-J.; Chen, C.-Y.; Chiou, L.-H; Wang, S.-J.; Dai, F.; Hsieh, T.; Tsai, R.-Y; Huang, D.; Ho, F. C.; Sol.-State. Electron. 2002, 46, 477 